\title{
Protracted Synaptogenesis after Activity-Dependent Spinogenesis in Hippocampal Neurons
}

\author{
U. Valentin Nägerl, ${ }^{1}$ German Köstinger, ${ }^{2}$ John C. Anderson, ${ }^{2}$ Kevan A. C. Martin, ${ }^{2}$ and Tobias Bonhoeffer ${ }^{1}$ \\ ${ }^{1}$ Max Planck Institute of Neurobiology, 82152 München-Martinsried, Germany, and ${ }^{2}$ Institute for Neuroinformatics, University of Zürich and \\ Eidgenössische Technische Hochschule Zürich, CH-8057 Zürich, Switzerland
}

\begin{abstract}
Activity-dependent morphological plasticity of neurons is central to understanding how the synaptic network of the CNS becomes reconfigured in response to experience. In recent years, several studies have shown that synaptic activation that leads to the induction of long-term potentiation also drives the growth of new dendritic spines, raising the possibility that new synapses are made. We examine this directly by correlating time-lapse two-photon microscopy of newly formed spines on CA1 pyramidal neurons in organotypic hippocampal slices with electron microscopy. Our results show that, whereas spines that are only a few hours old rarely form synapses, older spines, ranging from 15 to $19 \mathrm{~h}$, consistently have ultrastructural hallmarks typical of synapses. This is in agreement with a recent in vivo study that showed that, after a few days, new spines consistently form functional synapses. In addition, our study provides a much more detailed understanding of the first few hours after activity-dependent spinogenesis. Within tens of minutes, physical contacts are formed with existing presynaptic boutons, which slowly, over the course of many hours, mature into new synapses.
\end{abstract}

Key words: synaptic plasticity; two-photon imaging; correlated electron microscopy; theta-burst stimulation; LTP; spines

\section{Introduction}

The striking ability of the brain to learn and retain information is thought to rely primarily on activity-dependent synaptic plasticity. Whereas short-term information storage of a few hours duration is usually explained in terms of acute changes in the strength of synaptic connections that occur with long-term potentiation (LTP), long-term memory is thought to involve additional plastic changes in the "hardwiring," or the physical connectivity of neuronal circuits (Ramon y Cajal, 1894). Advances in time-lapse imaging techniques have enabled several groups to shed new light on the link between functional and morphological plasticity. The outgrowth of new spines or filopodia within minutes of LTP-inducing stimulation has been a consistent finding (Engert and Bonhoeffer, 1999; Maletic-Savatic et al., 1999; Jourdain et al., 2003; Nägerl et al., 2004). Because dendritic spines are the site of most excitatory synapses in the CNS, this is thought to be one important mechanism whereby functional changes induced by activity are made more permanent.

However, little is known about how the growth of new spines induced by synaptic activation is coupled to the formation of new synapses. A recent combined electron microscopic/in vivo imaging study by Knott et al. (2006) showed that spines that were generated in an experience-dependent paradigm and that persisted for a few days consistently formed synapses. In an electron

Received Feb. 5, 2007; revised May 18, 2007; accepted June 16, 2007.

We thank Rafael Yuste, Tom Mrsic-Flögel, and Corette Wierenga for comments on this manuscript, and Marianne Braun for technical help. We acknowledge the collaboration of Biolmaging Zentrum (Ludwig-Maximilians University Munich), T.I.L.L. Photonics, and the Max Planck Institute of Neurobiology.

Correspondence should be addressed to U. Valentin Nägerl, Max Planck Institute of Neurobiology, Am Klopferspitz 18,82152 München-Martinsried, Germany. E-mail: naegerl@neuro.mpg.de.

D0I:10.1523/JNEUROSCI.0511-07.2007

Copyright $\odot 2007$ Society for Neuroscience $\quad$ 0270-6474/07/278149-08\$15.00/0 microscopic study using the precipitation of $\mathrm{Ca}^{2+}$ to identify activated synapses, LTP induction was shown to lead to a transient increase in perforated synapses followed by a persistent increase in the frequency of boutons forming synapses with more than one spine, suggesting that LTP is associated with the formation of new synapses (Toni et al., 1999).

Although there is a broad consensus that spines that form as a result of neuronal activity are likely to become part of synapses, important details about the temporal and spatial dynamics of spine-based activity-dependent synaptogenesis have remained unresolved. Here we set out to examine directly the relationship between spinogenesis induced by a well defined stimulus and the ensuing synaptogenesis with a temporal resolution on the order of hours. We filled single CA1 pyramidal neurons in organotypic hippocampal slices with calcein dye to image the growth of new spines after local electrical theta-burst stimulation (TBS) of afferent fibers using time-lapse two-photon laser-scanning microscopy. Neurons were also filled with biocytin to produce an electron-dense label, which allowed us to correlate the fluorescence images with three-dimensional (3D) reconstructions based on serial section electron microscopy (EM) of the dendritic sites of spinogenesis.

We first show that TBS induced the growth of spines that persist for at least $1 \mathrm{~d}$, the longest time period we imaged. We then generated a population of newly formed spines that differed in the time that had passed between their generation and the fixation of the tissue. This allowed us to relate retrospectively the age of identified, newly formed spines with their synaptic status as judged by the ultrastructure of the surrounding neuropil. We found that the majority of new spines were in physical contact with a synaptic bouton, which almost invariably formed a synapse with another spine. However, despite their physical contact 
with these boutons, young spines (a few hours old) showed no clear evidence of synaptic structures, whereas older spines (15-19 $\mathrm{h}$ old) did. Thus, whereas LTP-inducing stimuli rapidly induce new spines in close proximity to existing boutons, synapse formation, as based on ultrastructural criteria, takes many hours.

\section{Materials and Methods}

Organotypic hippocampal slice cultures and recording solutions. Hippocampal slices (300 $\mu \mathrm{m}$ thick) from postnatal day 5-7 wild-type C57BL/6J mice were prepared, embedded in a plasma clot on glass coverslips, and incubated up to 2 weeks in a roller incubator at $35^{\circ} \mathrm{C}$, according to the Gähwiler method (Gähwiler, 1981). The age of the slice cultures for the experiments ranged between 7 and $13 \mathrm{~d}$ in vitro after the preparation. For the experiments, cultures were transferred into a recording chamber, in which they were continuously perfused with carbogenated $\left(95 \% \mathrm{O}_{2}, 5 \% \mathrm{CO}_{2}\right)$ artificial CSF containing the following (in mм): $126 \mathrm{NaCl}, 2.5 \mathrm{KCl}, 2.8 \mathrm{CaCl}_{2}, 0.5 \mathrm{MgCl}_{2}, 10$ glucose, 1.25 $\mathrm{NaH}_{2} \mathrm{PO}_{4}, 26 \mathrm{NaHCO}_{3}, 0.05$ glycine, and 1 pyruvate. The temperature was maintained at $35^{\circ} \mathrm{C}$, and the $\mathrm{pH}$ was 7.4.

Electrophysiology. Patch pipettes were used for electrical stimulation. They were filled with $3 \mathrm{M} \mathrm{NaCl}$ and $10 \mathrm{~mm}$ of the fluorescent dye calcein immobilized in agar. A chlorided silver wire was used to pass brief current pulses $(0.2 \mathrm{~ms})$ of $15-30 \mu \mathrm{A}$ from a stimulus isolator (World Precision Instruments, Berlin, Germany) through the patch pipette. TBS consisted of five trains ( $200 \mathrm{~ms}$ intertrain interval) of six pulses delivered at $100 \mathrm{~Hz}$, repeated five times every $10 \mathrm{~s}$. The tip of the electrode was positioned in the vicinity of the section of apical dendrite that was selected for time-lapse imaging. The minimal distance between the tip of the electrode and the dendrite was kept between 10 and $20 \mu \mathrm{m}$. In separate experiments, we verified that this arrangement of stimulus electrode and stimulation strength reliably produced subthreshold synaptic potentials, which were not contaminated by direct stimulation of voltage-gated conductances in the postsynaptic cell.

Two-photon light microscopy. Time-lapse two-photon laser-scanning microscopy (two-photon microscopy) was used to image over time the dendritic morphology of CA1 pyramidal neurons briefly ( $<2 \mathrm{~min}$ ) cofilled via a patch pipette with calcein ( $5 \mathrm{~mm}$ inside pipette) for fluorescence and biocytin (1 mm inside pipette) to produce an electron-dense label. The red excitation light ( $\lambda$ of $810 \mathrm{~nm}$ ) from a $5 \mathrm{~W}$ Mira-Verdi laser system (Coherent, Santa Clara, CA) was beam expanded sixfold using a Kepler telescope and routed through a Yanus 2 scanner (T.I.L.L. Photonics, Gräfelfing, Germany), a suitable dichroic mirror (LOT-Oriel, Darmstadt, Germany), and a $40 \times, 1.2$ numerical aperture, coverslip corrected water immersion objective (Zeiss, Oberkochen, Germany) mounted on an inverted IX70 microscope (Olympus Optical, Tokyo, Japan). The power of the excitation light could be adjusted continuously by an acusto-optical modulator (Polytec, Waldbronn, Germany), and its average value at the objective was set to $10-20 \mathrm{~mW}$. The fluorescence was detected by an external photomultiplier tube (R6357; Hamamatsu, Herrsching, Germany). Image acquisition and on-line analysis was performed by custom-made scanning software written in LabVIEW (National Instruments, Austin, TX), and image stacks were saved to disk for off-line analysis. The nominal image resolution was $115 \mathrm{~nm} /$ pixel in $x-y$. A piezoelectrical actuator (Physik Instrumente, Karlsruhe, Germany) was used to move the objective in the $z$-axis $(\Delta z=0.4 \mu \mathrm{m})$. Two or three stacks were acquired every $30 \mathrm{~min}$ before the stimulation and four to seven stacks after the stimulation at variable time intervals. Repeated acquisition of high-resolution image stacks allowed us to remove possible falsepositive events of spine creation or disappearance associated with the limited $z$-axis resolution of the microscope and spine rotational artifacts. A reference image was taken at the start of the experiment to facilitate alignment and to keep sample drift over the $24 \mathrm{~h}$ of the experiment at a minimum.

Two-photon microscopy image analysis. $4 \mathrm{D}(x, y, z, t)$ image stacks were processed and analyzed using the Imaris 4 software (Bitplane, Zürich, Switzerland). Individual stacks were spatially filtered by an edgepreserving algorithm, rescaled, and baseline subtracted. To facilitate overview and for display in the figures, the 3D stacks were volume ren- dered as 2D images using the blending projection view in Imaris. All image analysis was done by visual inspection of the individual image sections, as well as the rendered $2 \mathrm{D}$ projections of the image stacks.

Serial section electron microscopy and $3 D$ reconstructions. After the last time-lapse time point, slices were initially transferred into $35^{\circ} \mathrm{C}, 0.1 \mathrm{M}$ phosphate buffer (PB)-based fixative containing $4 \%$ paraformaldehyde, $15 \%$ picric acid, and $0.5 \%$ glutaraldehyde and subsequently stored at $4^{\circ} \mathrm{C}$ for $4 \mathrm{~h}$ on a shaker. Slices were removed from the coverslip using a paint brush, transferred though an ascending gradient of PB-based sucrose solutions, and then subjected to a freeze-thaw cycle using liquid nitrogen. The biocytin label was revealed using a Vectastein Elite ABC kit (Axxora, Grünberg, Germany) and 3,3-diaminobenzidenetetrahydrochloride (DAB) histology. Briefly, slices were incubated in $\mathrm{ABC}$ solution overnight before the peroxidase reaction end product was revealed by $\mathrm{DAB}$. To enhance the contrast for the EM, the slices were briefly treated with osmium tetroxide solution (4\%), followed by an uranylacetate $(1 \%)$-containing ethanol solution (\%70) for dehydration. The slices were embedded in Durcupan ACM resin (Fluka, Buchs, Switzerland). Sections were prepared for light microscopy, and regions of interest defined by the two-photon microscopy imaging were then observed under transmission EM (for details, see Anderson et al., 1994). Briefly, serial ultrathin sections were collected at $60 \mathrm{~nm}$ thickness on Pioloformcoated single-slot copper grids (Bio-Rad, Hempstead, UK). Labeled spines were photographed at a magnification of $25,000 \times$, using an analog camera to facilitate observing the postsynaptic densities. Digitized images were processed using the Reconstruct program (John C. Fiala, Boston University, Boston, MA) to outline the labeled structures for 3D reconstructions, which facilitated relating the EM data with the two-photon images. For the figures, the $3 \mathrm{D}$ outlines were rendered using the Blender software version 2.4. (open source shareware, www.blender.org).

EM image analysis. Newly formed spines were re-identified using serial-section EM and 3D reconstructions. During re-identification, all relevant EM sections were inspected, and the presence or absence of the following ultrastructural characteristics classically associated with mature synapses was scored: (1) boutons abutting the spine of interest, (2) distinct clusters of vesicles therein, and (3) a synaptic cleft. A "1" was assigned if the structure was present, otherwise a " 0 " was assigned. For the synaptic cleft, we subdivided the score into four levels $(0,0.25,0.75$, and 1), reflecting the wider range in the visibility of a widening between the presynaptic and postsynaptic membranes typical of a synaptic cleft. Statistical comparison of the synaptic score parameters of the control, young, and old spine groups was done using the Mann-Whitney $U$ test in the Statistica software (StatSoft, Hamburg, Germany). All values shown are mean $\pm \mathrm{SE}$, and $p$ values are two sided. In total, 22 newly formed spines and 39 preexisting spines were reconstructed in three dimensions and analyzed.

\section{Results}

We applied theta-burst stimulation to produce synaptic strengthening and the associated growth of new spines on dendrites of CA1 pyramidal neurons in organotypic hippocampal slice cultures as described previously (Nägerl et al., 2004). Using timelapse two-photon microscopy (Denk et al., 1990), we monitored dendritic morphology and spine growth on CA1 pyramidal neurons (Fig. 1) for $30 \mathrm{~min}$ up to $19 \mathrm{~h}$, before fixing the slices. The spines were subsequently re-identified using correlated light and serial section electron microscopy, and the neuropil surrounding the newly formed spines was analyzed to identify possible ultrastructural indicators of new synapses. This allowed us to address important aspects of when and how spines that are generated in an activity-dependent paradigm form synapses.

\section{TBS induces the growth of new spines that are stable}

To study the relationship between spinogenesis and synaptogenesis, we first examined the temporal stability of spines that form de novo as a result of synaptic stimulation. We performed time- 


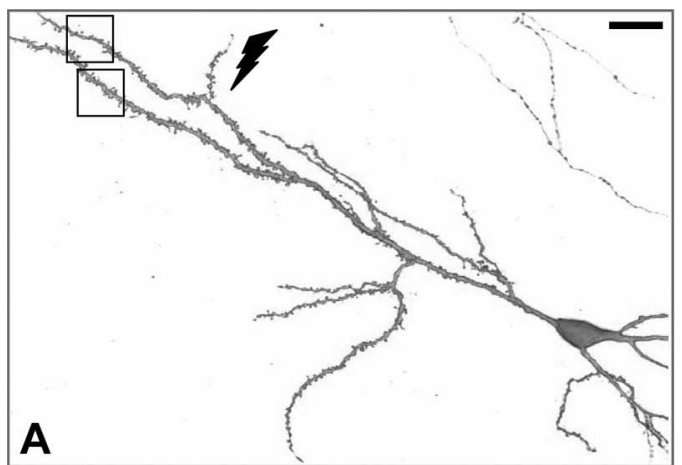

B
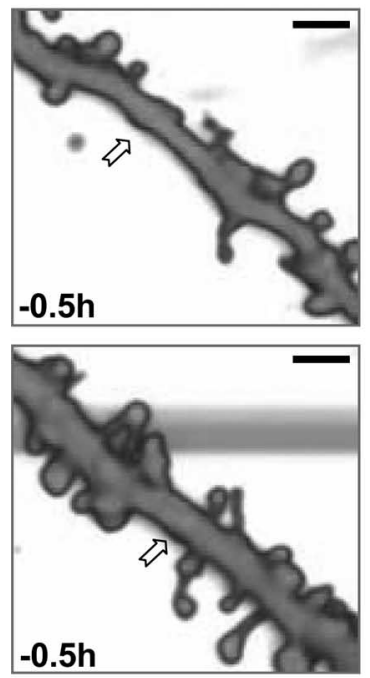

$+2 \mathrm{~h}$
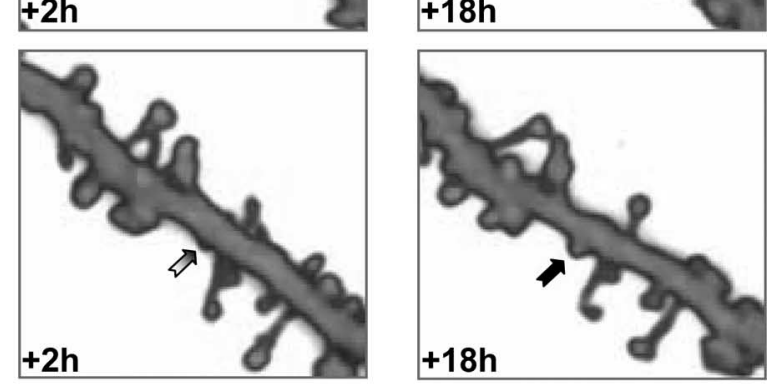

C
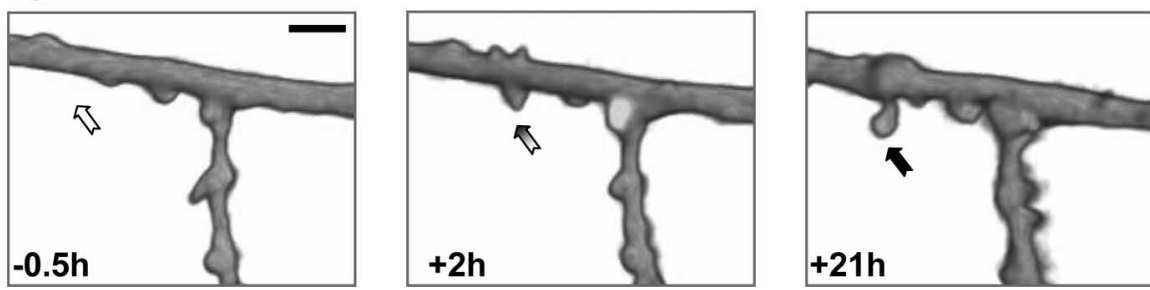

Figure 1. Theta-burst stimulation leads to growth of persisting dendritic spines on pyramidal neurons. $A$, Two-photon microscopy image of a calcein-filled CA1 pyramidal neuron stimulated by TBS at the site indicated by the arrow. Scale bar, $20 \mu \mathrm{m}$. Rectangles indicate dendritic section of interest imaged at $4 \times$ zoom in a time-lapse series shown. $\boldsymbol{B}$, Indicated times are relative to time point of TBS stimulation. Arrows indicate newly growing spines, the open/filled symbols indicating absence/presence. $\boldsymbol{C}$, An example from a different experiment. Scale bars, $2 \mu \mathrm{m}$.

lapse imaging for up to $1 \mathrm{~d}$, which allowed us to quantify how many of the newly formed spines wither away or survive until fixation. Figure 1, $B$ and $C$, shows examples of spinogenesis after TBS. The newly formed spines shown were imaged for at least $16 \mathrm{~h}$. The vast majority of spines that grew after TBS persisted beyond the last imaging time point, after which the slices were fixed within $30 \mathrm{~min}$. Supplemental Figure 1 (available at www. jneurosci.org as supplemental material) provides an overview of the spines that grew and persisted or disappeared over the course of our time-lapse imaging experiments, indicating that, first, TBS indeed induces the growth of spines that, second, are predominantly long lasting. This is consistent with our previous study (Nägerl et al., 2004), which showed that TBS significantly raises the rate of spinogenesis over unstimulated control conditions, which under our experimental conditions is negligible.

\section{Most preexisting spines carry mature synapses}

To provide a basis of comparison for the newly formed spines and to estimate the fraction of preexisting spines that carry ultrastructurally mature synapses, we analyzed at the EM level spines that were present throughout the imaging experiments. We scored the visibility of structures that are commonly used as ultrastructural criteria for synapses, namely a presynaptic bouton, synaptic cleft, and clusters of vesicles at the point of contact between bouton and spine. Postsynaptic densities were oftentimes obscured by the reaction end product. Figure 2 illustrates the approach of re-identifying specific spines that could be seen under time-lapse two-photon microscopy using serialsection EM. Figure 2, $A$ and $B$, shows the same section of dendrite imaged $19 \mathrm{~h}$ apart. Figure $2 C$ shows the same section of dendrite after histological processing to visualize the biocytin at the light microscopic level. Spines could be readily reidentified after fixation of the tissue, indicating that fixation-related artifacts did not pose a serious problem. Similarly, the overall structure was well preserved after ultrathin sectioning, as the reconstruction of serial sections in Figure $2 D$ shows. The EM micrographs in Figure 2, $E$ and $F$, shows sections through the heads of the spines indicated by the numbers in the reconstruction. These spines form synapses as judged by the presence of a synaptic cleft and a bouton filled with vesicles. Figure 2, $G$ and $H$, shows additional examples of preexisting spines that reveal ultrastructural evidence for synapses (supplemental Fig. $A 1, A 2$, available at www.jneurosci.org as supplemental material).

The vast majority of preexisting, or "control," spines were from serial sections of dendrite adjacent to the new spines. They showed clear ultrastructural evidence that they formed synapses with a bouton, in keeping with the literature (White and Hersch, 1982; Mates and Lund, 1983; Sorra and Harris, 1998; Kirov et al., 1999). For a quantitative comparison, we scored whether or not (1) a presynaptic bouton, (2) a synaptic cleft, and (3) clusters of vesicles could be seen in any of the EM sections (for details, see Materials and Methods). For the control spines, the scores were consistently high, as shown in Table 1 for eight examples arbitrarily selected from a total of 39 analyzed preexisting spines. A total of $77 \%$ of the control spines showed definitive evidence of a mature synapse (all scores are 1), whereas $10 \%$ failed to show evidence (synaptic cleft score is 0 ), and the remaining $13 \%$ showed partial evidence (synaptic cleft score is either 0.25 or 0.75 , all other scores being 1 ).

To check the reliability of our method and its ability to recognize synapses also on small spines, we examined the synaptic scores of a random subset of control spines, whose volumes var- 

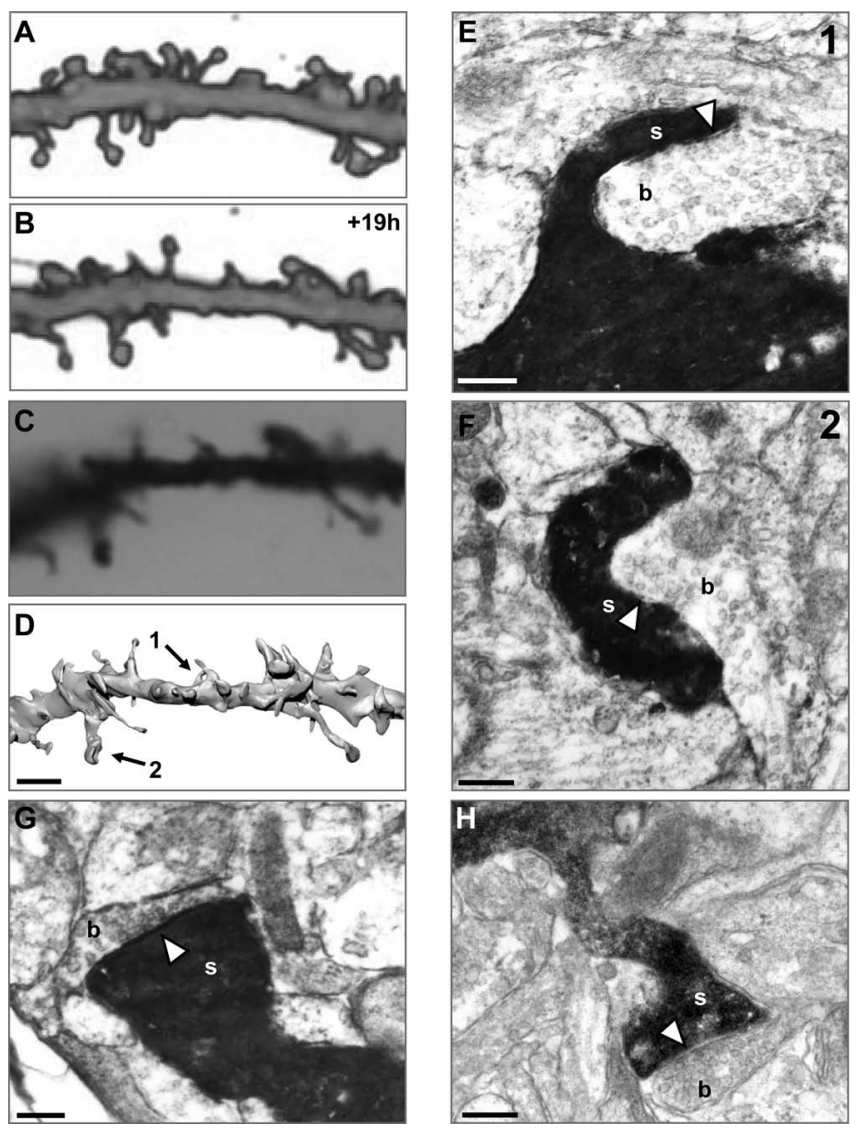

Figure 2. Most preexisting spines are part of ultrastructurally mature synapses. $A$, An unstimulated section of dendrite. $\boldsymbol{B}$, The same section imaged $19 \mathrm{~h}$ later. $\boldsymbol{C}$, A light microscopic image of the same section of dendrite, after fixation and DAB reaction. $D, 3 D$ reconstruction of the same dendrite based on serial-section EM. Scale bar: $\boldsymbol{A}-\boldsymbol{D}, 2 \mu \mathrm{m} . \boldsymbol{E}, \boldsymbol{F}$, Electron micrographs of labeled spines (s) marked by the arrows in $\boldsymbol{D}$. Presynaptic boutons (b) separated by synaptic clefts (white arrowheads) are clearly visible. $\mathbf{G}, \boldsymbol{H}$, Two additional examples of electron micrographs through preexisting spines (supplemental Fig. 2A1,A2, available at www.jneurosci.org as supplemental material). Scale bars in EM sections, $200 \mathrm{~nm}$.

ied widely (from 0.0289 to $0.3146 \mu \mathrm{m}^{3}$ with a mean of $0.1421 \pm$ $\left.0.0240 \mu \mathrm{m}^{3} ; n=14\right)$. The fact that we could recognize synapses on all spines, even the very smallest ones (for data on each control spine listed, see Table 1), indicates that our method was not biased toward detecting synapses only on bigger spines.

Young spines are in contact with boutons but do not form synapses

We first performed our analysis on newly formed "young" spines, defined as ranging from $30 \mathrm{~min}$ to $8 \mathrm{~h}$ in age. Figure $3, A$ and $B$, shows time-lapse two-photon microscopy images of a section of dendrite separated by $3 \mathrm{~h}$, the boxes marking sites of spinogenesis, shown with higher magnification in Figure 3, $C$ and $H$, and reconstructed in $D$ and $I$. The corresponding EM images in Figure 3, $F, G$, and $J$, illustrate that these young spines did not form any synapses, because no synaptic clefts or presynaptic specializations are visible in any of the other serial EM sections (typically $\sim 10$ sections). However, in most cases (in 9 of 13 young spines), presynaptic boutons could be seen in physical contact with the young spines, and most of these boutons formed synapses with other unlabeled targets (Fig. 3E, F, Table 1) (supplemental Fig. $2 B$, available at www.jneurosci.org as supplemental material).

The mean scores assigned for the population of young spines (aged $3.2 \pm 0.7 \mathrm{~h} ; n=13$ ) are consistently lower than for the
Table 1. Synaptic scores for young, old, and control spines

\begin{tabular}{|c|c|c|c|c|c|c|}
\hline Spine & Age (hour: & & Size $\left(\mu \mathrm{m}^{3}\right)$ & Boutons & Synaptic cleft & Vesicles \\
\hline N1 & | & 0.5 & 0.0306 & & & \\
\hline N2 & $\square$ & 1 & 0.1061 & & & \\
\hline N3 & $\rrbracket$ & 1 & 0.1685 & & C & \\
\hline N4 & $\square$ & 1 & 0.0224 & & C & \\
\hline N5 & 口 & 2 & 0.0367 & & & \\
\hline N6 & 口 & 2 & 0.0774 & & & \\
\hline N7 & 口 & 2 & 0.0541 & & & \\
\hline N8 & $\square$ & 3.5 & 0.0173 & & & \\
\hline N9 & $\square$ & 4 & 0.0382 & & & \\
\hline N10 & $\square$ & 4 & 0.0234 & & C & \\
\hline N11 & $\square$ & 4 & 0.0808 & & C. & \\
\hline N12 & L & 8 & 0.0174 & & C & \\
\hline N13 & \begin{tabular}{|l}
$\square$ \\
\end{tabular} & 8 & 0.0234 & & 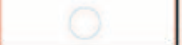 & \\
\hline N14 & [ & 15 & 0.2220 & & 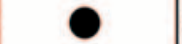 & \\
\hline N15 & [ & 15 & 0.0798 & & C & \\
\hline N16 & & 15 & 0.0718 & & C & \\
\hline N17 & & 16 & 0.1867 & & C & \\
\hline N18 & & 16 & 0.1388 & & C & \\
\hline N19 & & 17 & 0.1400 & & 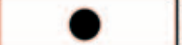 & \\
\hline $\mathrm{N} 20$ & & 17 & 0.1565 & & C & \\
\hline N21 & & 17 & 0.3331 & & 6 & \\
\hline N22 & 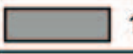 & 19 & 0.1527 & & $C$ & \\
\hline
\end{tabular}

\begin{tabular}{|c|c|c|c|}
\hline C1 & 0.0839 & 0 & 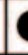 \\
\hline C2 & 0.0289 & 0 & 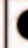 \\
\hline C3 & 0.0642 & 0 & 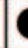 \\
\hline $\mathrm{C} 4$ & 0.0562 & (C) & \\
\hline C5 & 0.0628 & 0 & \\
\hline C6 & 0.1654 & 0 & \\
\hline C7 & 0.3146 & 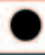 & \\
\hline C8 & 0.0682 & ค & \\
\hline
\end{tabular}

Symbolized are the scored synaptic parameters for all newly formed spines that were analyzed, ordered by age, and for a random subset of the preexisting spines. The length of the gray bar scales with the age of the spines; the black bar indicates preexisting spines. The column "Size" lists the volumes of the spines. In the column "Boutons," the circles indicate the number of boutons that were in contact with a new spine; no entry means that no bouton was seen in contact with the labeled spine. The oblique symbols indicate the presence of synapses formed by the boutons with other unlabeled targets. In the column "Synaptic cleft," the gray shading in the circles signifies the visibility of a synaptic cleft in the EM sections. In the column "Vesicle clusters," the filled circles indicate the number of vesicle clusters in the immediate vicinity of the new spine.

preexisting spines, as indicated in Table 1 (synaptic bouton, $0.85 \pm 0.22$ compared with $1.87 \pm 0.14, p=0.0006$; synaptic cleft, $0.13 \pm 0.08$ compared with $0.79 \pm 0.07, p<0.0001$; cluster of vesicles, $0.44 \pm 0.18$ compared with $1.15 \pm 0.06, p<0.0001$ ). In 9 of a total of 13 young spines analyzed, vesicle-filled boutons were in physical contact with the spine. In the remaining four cases, the spines were orphan and jutted out into regions of neu- 

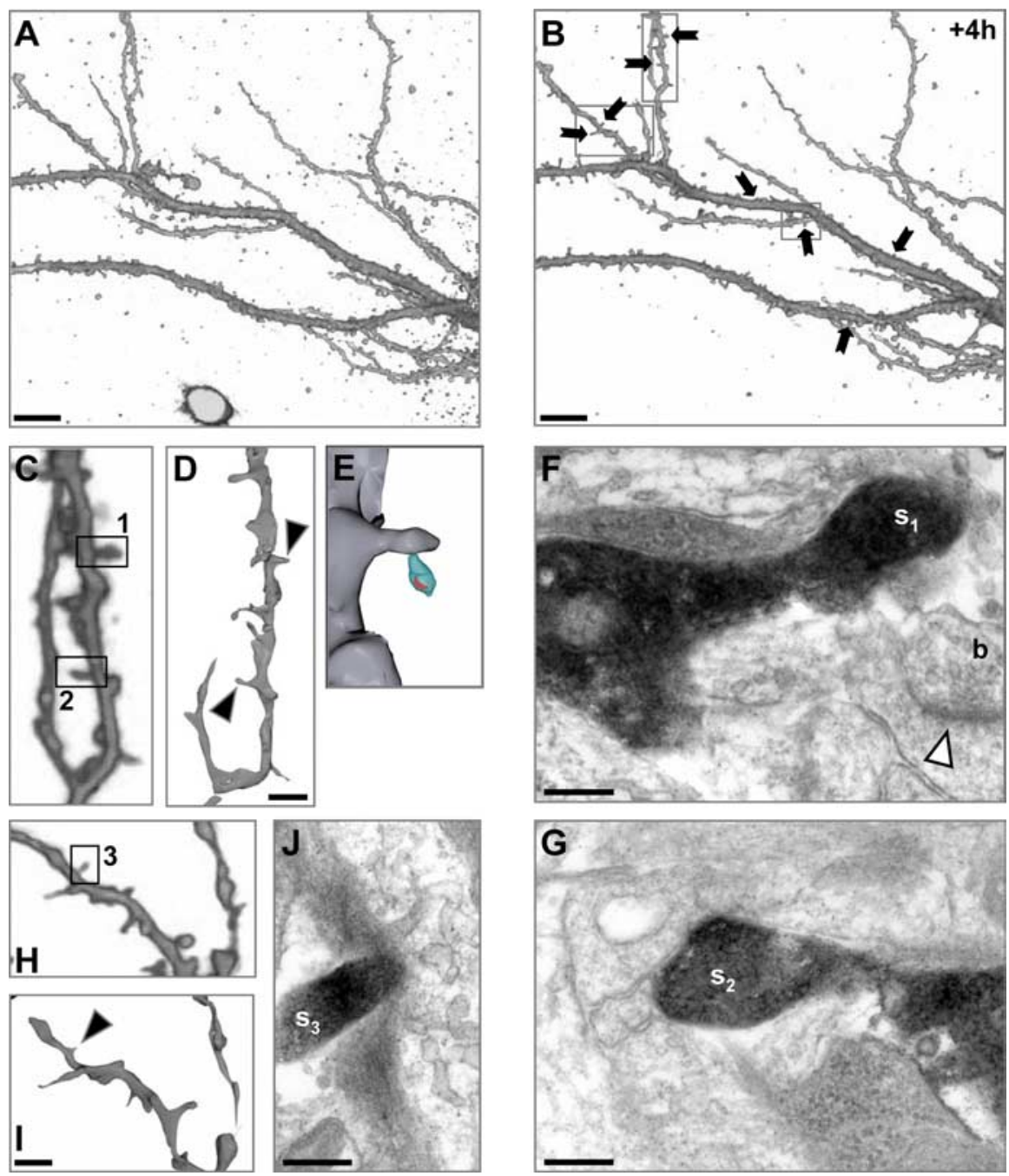

Figure 3. Younger spines are in physical contact with synaptic boutons but do not yet show ultrastructural signs of mature synapses. $\boldsymbol{A}, \boldsymbol{B}, \mathrm{A}$ stimulated section of dendrite imaged $4 \mathrm{~h}$ apart ( 3 of the sites of spinogenesis, marked by the arrows, are shown at greater magnification in $\boldsymbol{C}$ and $\boldsymbol{H}$ ) (supplemental Fig. $2 B$, available at www.jneurosci.org as supplemental material). $\boldsymbol{D}, \boldsymbol{I}$, The 3 reconstructions of these regions. Scale bars: $\boldsymbol{C}, \boldsymbol{D}, \boldsymbol{H}, \boldsymbol{I}, 2 \mu \mathrm{m} . \boldsymbol{F}, \boldsymbol{G}, \boldsymbol{J}$, Electron micrographs of the labeled spines (indicated by the numbers), illustrating that these newly formed young spines were not associated with a synaptic cleft or a bouton (b) with clustered vesicles next to the point of contact with the labeled spine (s) (supplemental Fig. $2 B$, available at www.jneurosci.org as supplemental material). Scale bars in EM sections, $200 \mathrm{~nm}$. $\boldsymbol{E}$, The labeled spine 1 was in contact with a presynaptic bouton (in blue) that formed a synapse (in red) with another unlabeled target (supplemental Fig. 2B3, available at www.jneurosci.org as supplemental material).

ropil in which there were no boutons. The mean volume of these young new spines was significantly smaller than the volume of the control spines (young spines, $0.0534 \pm 0.0124, n=13$; control spines, $\left.0.1421 \pm 0.0240 \mu \mathrm{m}^{3}, n=14 ; p=0.0036\right)$.

To estimate the likelihood of a spine contacting a presynaptic bouton by chance, we measured the fractional areas in a given field of view occupied by all boutons. This area was on average $9.1 \pm 0.4 \%$ ( $n=10$ fields of view). If a spine were considered as a geometrical point, this would mean that by chance $\sim 1$ in 10 spines should come into contact with a preexisting bouton (in 2D). Considering that the fractional area of the newborn spine comprised $\sim 1 \%$ in the measured fields of view, the fact that 9 of 13 newly formed spines are in physical contact with boutons indicates that there is a directed growth or stabilization process for the newly formed spines.

The fractional area of the entire axon amounts to $\sim 30 \%$ (Braitenberg et al., 1998). Therefore, even under the assumption that the entire axon is competent to form synapses, the number of chance encounters between newly formed spines and boutons would still only be 4 of the total of 13.

\section{Older spines bear ultrastructural features of mature synapses}

A few hours after the spines were formed, none formed synapses despite the fact that most of them were in contact with presynaptic boutons. This clearly indicated that synapse maturation is not a rapid process but requires considerably more than a few hours. To determine how much time, we extended the duration of the time-lapse two-photon microscopy experiment to generate a population of TBS-induced, newly formed spines that were approximately five times older on average than the young spines (old spines, $16.3 \pm 0.4 \mathrm{~h}, n=$ 9; young spines, $3.2 \pm 0.7 \mathrm{~h}, n=13$ ).

Figure 4 shows time-lapse two-photon microscopy images of a dendritic section before $(A)$ and after $(B, C)$ TBS with two newly formed spines, aged 16 and $17 \mathrm{~h}$ at the end of the experiment. The same section of dendrite was reconstructed based on the serial-section EM data, and the newly formed spines detected in the twophoton microscopy experiment could readily be re-identified (Fig. $4 D$ ). The corresponding EM sections of the two newly formed spines reveal ultrastructural characteristics indicative of mature synapses. We analyzed a total of nine old spines, ranging from 15 to $19 \mathrm{~h}$ in age, and assigned the synaptic scores using the same criteria as for the young and control spines. The mean synaptic scores for the old spines are significantly higher than for the young spines (synaptic bouton, $1.78 \pm$ 0.22 compared with $0.85 \pm 0.22, p=$ 0.0096 ; synaptic cleft, $0.58 \pm 0.19$ compared with $0.13 \pm 0.08, p=0.0027$; cluster of vesicles, $1.22 \pm 0.22$ compared with $0.38 \pm 0.14, p=0.0032)$ and statistically not distinguishable from the control spines $(p>0.15)$ (Fig. $5 A)$. This suggests that the old spines were markedly further along in synaptic development than the young spines, and indeed some formed synapses, because their synaptic scores closely resemble those of the preexisting spines. This conclusion is consistent with the morphometric analysis, which shows that the old spines had volumes that were significantly larger than the young spines but that were indistinguishable from the control spines (old, $0.1646 \pm 0.0262$, $n=9$; young, $0.0534 \pm 0.0124, n=13, p<0.001$; control spines, $\left.0.1421 \pm 0.0240 \mu \mathrm{m}^{3}, n=14, p=0.55\right)$.

\section{Discussion}

Our study addresses the long-standing question of how activityinduced spinogenesis in mammalian CNS neurons relates to synaptogenesis. Although numerous recent time-lapse imaging studies have reported the outgrowth of new spines or filopodia 

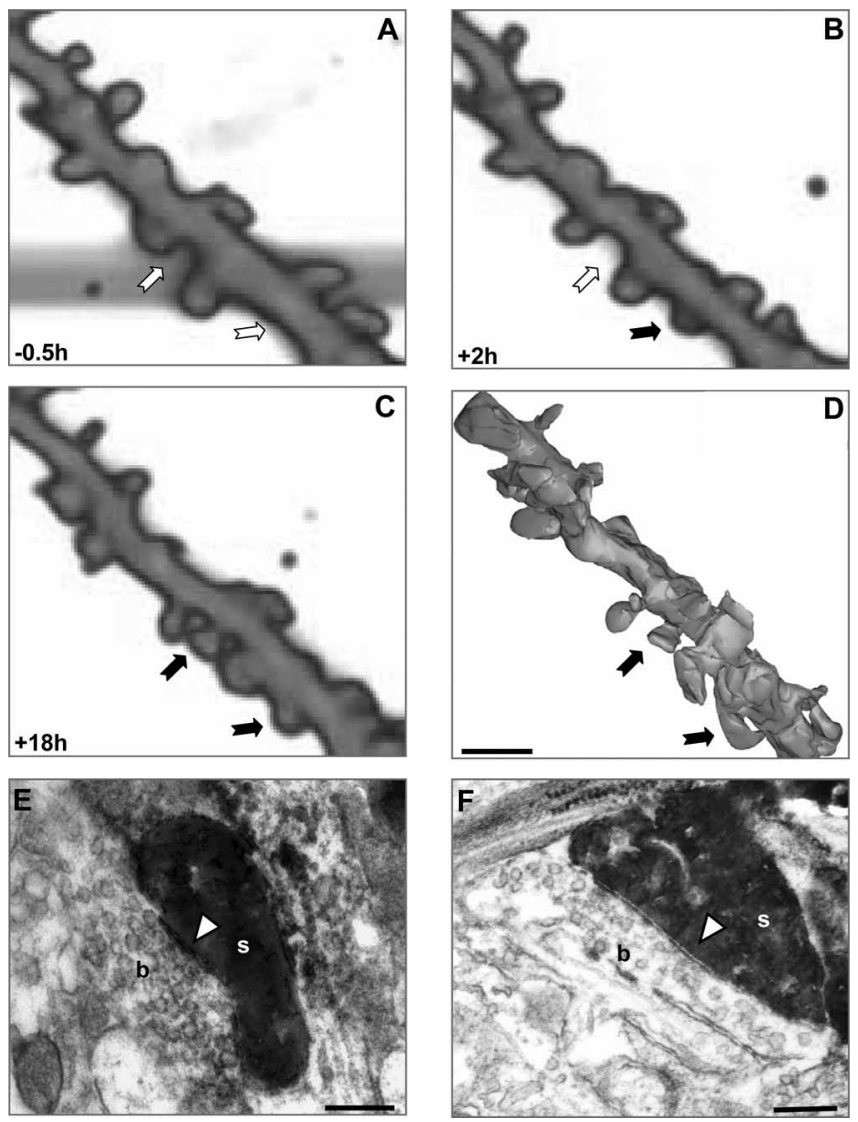

Figure 4. Older spines show ultrastructural signature of mature synapses. $A-C$, Time-lapse images of a stimulated section of dendrite; the arrows indicate two growing spines (indicated times are relative to time point of TBS stimulation). $\boldsymbol{D}, 3 \mathrm{D}$ reconstruction based on serial-section EM of same section of dendrite, with arrows pointing at the spines that grew and lived to 16 and $17 \mathrm{~h}$, respectively. Scale bar: $\boldsymbol{A}-\boldsymbol{D}, 2 \mu \mathrm{m} . \boldsymbol{E}, \boldsymbol{F}$, Electron micrographs of the newly grown, labeled spines (s), revealing ultrastructural signs associated with maturity, such as a bouton (b) filled with vesicles and a synaptic cleft, indicated by the white arrowhead (supplemental Fig. 2C, available at www.jneurosci.org as supplemental material). Scale bars in EM sections, $200 \mathrm{~nm}$.

after LTP-inducing stimulation (Engert and Bonhoeffer, 1999; Maletic-Savatic et al., 1999; Toni et al., 1999; Jourdain et al., 2003; Nägerl et al., 2004), it is still not known whether newly formed spines form synapses and how they integrate into the existing synaptic network. Here, we examined the temporal relationship between activity-dependent spinogenesis and synaptogenesis in CA1 pyramidal neurons in organotypic hippocampal slice cultures using time-lapse two-photon microscopy followed by correlated serial-section EM on identified, newly formed spines. Given that the rate of spontaneous or developmental spinogenesis even at the relatively young age of the tissue used in the experiments is negligible (Nägerl et al., 2004), virtually all new spines we analyzed are bound to be a result of the plasticity paradigm. These new spines, soon after their initial appearance, come into physical contact with presynaptic boutons, but they do not make synapses within the first few hours after spinogenesis. Within 15-19 h, however, most of them form synapses as judged by ultrastructural criteria.

The timeline of contact formation between spines and synaptic boutons in our experiments suggests a prolonged, multistep process for synaptogenesis, consistent with the model for developmental synaptogenesis advanced by Stephen Smith and coworkers (Dailey and Smith, 1996; Ziv and Smith, 1996), whereby a filopodium first grows out to or "captures" an axon and then converts into a spine as synaptogenesis ensues. Whereas we rarely observed the growth of filopodia-like structures, our data suggests that the postsynaptic spine indeed directs its growth toward a preexisting bouton to establish a physical contact before forming, over a period of $1 \mathrm{~d}$ or so, a synapse with the bouton, possibly leading to the removal of the preexisting synapse formed between that bouton and an unlabeled spine (Fig. 5B).

This interpretation is supported by several lines of evidence. The finding that young spines consistently fail to form synapses, despite being in physical contact with synaptic boutons almost all of which form synapses with other targets, is evidence that normally a shaft synapse is not converted to a spine synapse by budding from a dendrite. Although a similar scenario was suggested in a recent paper (Knott et al., 2006), the fact that our data follow the transition from a mere physical to a mature synaptic contact provides more direct proof for it.

We observed that the fraction of boutons that form synapses with other targets is highest for the young spines (7 of 9, or 78\%), intermediate for old spines ( 4 of 9 , or $44 \%$ ), and lowest for control spines ( 5 of 14 , or $36 \%$ ) (Table 1), raising the possibility that the boutons contacted by new spines are transiently innervated by multiple spines, eventually losing the "excess" synapses or splitting into two. More detailed and longer-lasting time-lapse studies will be necessary to study this in more detail.

Many immunohistochemical studies have shown the close proximity of synapsin punctae with postsynaptic structures and taken that as evidence for functional synaptic contacts. Indeed, we also observed that for young spines (data not shown). However, the present data show that this must not be (mis)taken as definitive proof. The resolution of light microscopic data can simply not distinguish whether presynaptic boutons form a synapse with the spine of interest or an adjacent unlabeled spine.

Given that many more new spines end up in contact with a bouton than would be predicted assuming random outgrowth, it seems likely that new spines grow out in a directed manner and become selectively stabilized adjacent to presynaptic boutons. A recent study by Richards et al. (2005) showed that protrusions grow out from dendritic spines on CA1 pyramidal neurons toward sources of locally applied glutamate, raising the possibility that new contact formation relies on new spines being guided to their presynaptic target along local glutamate gradients in which they become stabilized in a glutamate-dependent process (Fischer et al., 2000). In the alternative scenario, the bouton could grow toward the shaft of the dendrite, in which it induces a spine. This, however, would require an extensive reorganization of both presynaptic and postsynaptic elements, making this a less likely scenario. Indeed, our evidence that the boutons contacted by the new spines already have mature synapses implies that the bouton is in a stable position and the new synapses are attributable to the spine growing toward a likely partner. This interpretation is in line with the conclusions by a recent study (Knott et al., 2006), which also found that new spines tend to be preferentially contacted by boutons with multiple synapses (multiple-spine boutons), whereas control spines are typically innervated by singlespine boutons. In fact, both studies draw similar overall conclusions: essentially that new spines form new synapses on preexisting boutons but do so for completely different model systems, suggesting that "bouton targeting" may represent a general strategy used by newly emergent spines. Although the study by Knott et al. was performed in vivo, our study has the benefit of relying on a classical plasticity paradigm. Most importantly, however, because of the higher temporal resolution, our study sheds light on the critical, early time right after the spines have formed. 
A

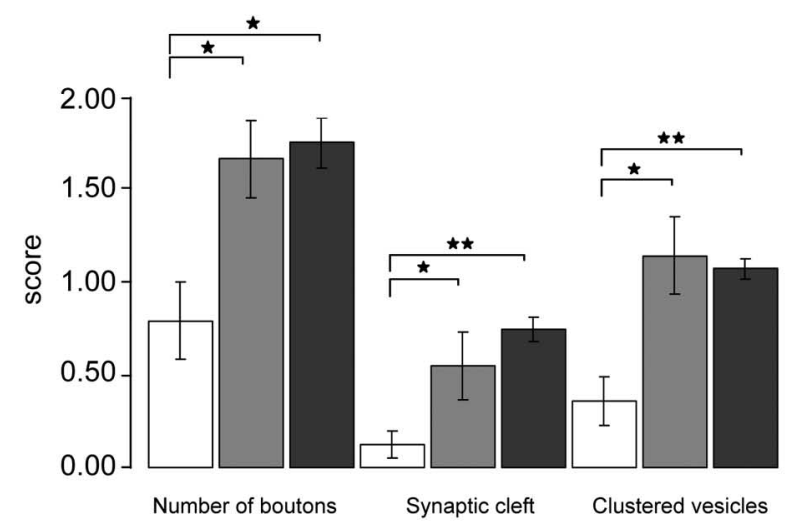

$\square$ young spines (1-8 hours), $n=13$

$\square$ old spines (15-19 hours), $n=9$

- preexisting spines, $\mathrm{n}=39$

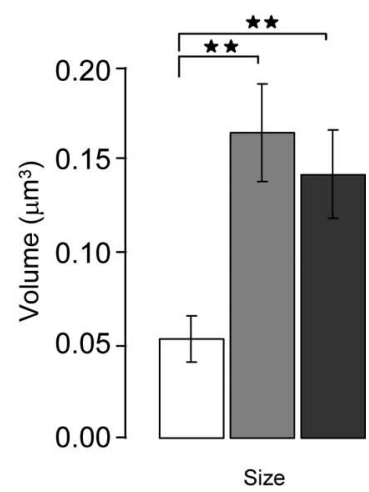

B
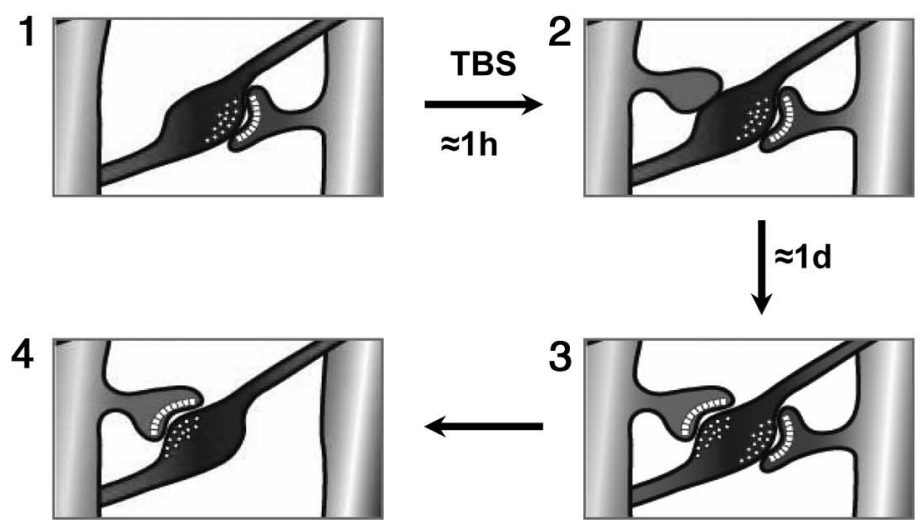

Figure 5. A, Statistics of synaptic parameters and volumes of young, old, and control spines. $B$, Model of spinogenesis and synaptogenesis. Scheme of a newly formed spine contacting a synaptic bouton. 1, Before TBS-induced spinogenesis; $2, \sim 1 \mathrm{~h}$ after TBS when the new spine gets in contact with a synaptic bouton; $3,1 \mathrm{~d}$ later, when synaptogenesis is complete; 4 , later on, one of the synapses formed by the new and the unlabeled spines with the preexisting bouton may be removed in a competitive manner. ${ }^{*} p<0.01 ;{ }^{* *} p<0.001$.

This warrants a number of new and important conclusions such as that new spines grow out toward preexisting boutons in a targeted manner and that multiple-spine boutons often are transient with the preexisting synapses being pruned as the new synapses mature.

It is clear from these data that spinogenesis, which can occur within minutes, and synapse formation, which takes hours, are distinctly different steps in spine-based synaptogenesis. This prolonged timescale of synapse formation is much longer than estimates from immunohistochemical studies, which indicate that synapses can be built within $2 \mathrm{~h}$ of axodendritic contact formation (Dailey and Smith, 1996; Friedman et al., 2000; Okabe et al., 2001). Rather, our data are consistent with older retrospective EM studies suggesting that synaptic junction development is a protracted process requiring up to $48 \mathrm{~h}$ (Rees et al., 1976; Cotman and Nietosampedro, 1984). However, these studies relied on developmental cell culture preparations, which are very different from our experiments in which we used an activity-dependent paradigm to drive spinogenesis in a more mature organotypic slice preparation. Whereas one might argue that the sequence of synaptogenic events is likely to be stereotypical, the time needed to assemble a synapse is likely to depend strongly on the developmental stage of the tissue and might very well be modulated by the level of background activity. Recent data from our group have demonstrated an important role for ongoing neuronal activity in consolidating the late phase of LTP (Fonseca et al., 2006), raising the possibility that the level of spontaneous neuronal activity influences the timeline of synaptogenesis.

Because we do not know whether the newly formed spines make contact with the activated presynaptic fibers, it is not clear whether the new synapses contribute to the synaptic enhancement that generated them. Given that it may take $\sim 1 \mathrm{~d}$ for newly formed spines to mature into synapses, clearly they do not support the synaptic enhancement that can be observed over the course of LTP experiments commonly performed in acute slice preparations. Rather, it is conceivable that the new synapses serve a more long-term or homeostatic function that allows the neuron to maintain its dynamic range for the ability to express synaptic enhancement at a later time. Our data does not allow us to distinguish whether the new synapses are indeed functional, or represent so-called "silent" synapses that lack AMPA receptor channels (Isaac et al., 1995; Liao et al., 1995; Durand et al., 1996). Moreover, it is conceivable that the nascent synapses are actually functional before the ultrastructural hallmarks have fully matured as discussed in the literature (Katz and Shatz, 1996; Ahmari and Smith, 2002). Approaches relying, for instance, on immunogold EM or $\mathrm{Ca}^{2+}$ imaging could in principle make these distinctions. Finally, our analysis was "spinocentric"; synaptogenic events that did not involve spine changes would have been missed, e.g., the growth of a new bouton toward a preexisting spine (but we consider this unlikely at least for the boutons that have mature synapses). As opposed to the plasticity of spines, we know very little about any changes occurring on the presynaptic side. The extent to which presynaptic elements participate in activity-dependent structural plasticity sorely needs to come into focus in future studies.

\section{References}

Ahmari SE, Smith SJ (2002) Knowing a nascent synapse when you see it. Neuron 34:333-336.

Anderson JC, Douglas RJ, Martin KA, Nelson JC (1994) Map of the synapses formed with the dendrites of spiny stellate neurons of cat visual cortex. J Comp Neurol 341:25-38.

Braitenberg V, Schèuz A, Braitenberg V (1998) Cortex statistics and geometry of neuronal connectivity. Berlin: Springer.

Cotman CW, Nietosampedro M (1984) Cell biology of synaptic plasticity. Science 225:1287-1294.

Dailey ME, Smith SJ (1996) The dynamics of dendritic structure in developing hippocampal slices. J Neurosci 16:2983-2994.

Denk W, Strickler JH, Webb WW (1990) 2-photon laser scanning fluorescence microscopy. Science 248:73-76.

Durand GM, Kovalchuk Y, Konnerth A (1996) Long-term potentiation and functional synapse induction in developing hippocampus. Nature 381:71-75. 
Engert F, Bonhoeffer T (1999) Dendritic spine changes associated with hippocampal long-term synaptic plasticity. Nature 399:66-70.

Fischer M, Kaech S, Wagner U, Brinkhaus H, Matus A (2000) Glutamate receptors regulate actin-based plasticity in dendritic spines. Nat Neurosci 3:887-894.

Fonseca R, Nägerl UV, Bonhoeffer T (2006) Neuronal activity determines the protein synthesis dependence of long-term potentiation. Nat Neurosci 9:478-480.

Friedman HV, Bresler T, Garner CC, Ziv NE (2000) Assembly of new individual excitatory synapses: time course and temporal order of synaptic molecule recruitment. Neuron 27:57-69.

Gähwiler BH (1981) Organotypic monolayer cultures of nervous tissue. J Neurosci Methods 4:329-342.

Isaac JTR, Nicoll RA, Malenka RC (1995) Evidence for silent synapses: implications for the expression of LTP. Neuron 15:427-434.

Jourdain P, Fukunaga K, Muller D (2003) Calcium/calmodulin-dependent protein kinase II contributes to activity-dependent filopodia growth and spine formation. J Neurosci 23:10645-10649.

Katz LC, Shatz CJ (1996) Synaptic activity and the construction of cortical circuits. Science 274:1133-1138.

Kirov SA, Sorra KE, Harris KM (1999) Slices have more synapses than perfusion-fixed hippocampus from both young and mature rats. J Neurosci 19:2876-2886.

Knott GW, Holtmaat A, Wilbrecht L, Welker E, Svoboda K (2006) Spine growth precedes synapse formation in the adult neocortex in vivo. Nat Neurosci 9:1117-1124.

Liao DZ, Hessler NA, Malinow R (1995) Activation of postsynaptically silent synapses during pairing-induced LTP in CA1 region of hippocampal slice. Nature 375:400-404.

Maletic-Savatic M, Malinow R, Svoboda K (1999) Rapid dendritic morpho- genesis in CA1 hippocampal dendrites induced by synaptic activity. Science 283:1923-1927.

Mates SL, Lund JS (1983) Neuronal composition and development in lamina-4C of monkey striate cortex. J Comp Neurol 221:60-90.

Nägerl UV, Eberhorn N, Cambridge SB, Bonhoeffer T (2004) Bidirectional activity-dependent morphological plasticity in hippocampal neurons. Neuron 44:759-767.

Okabe S, Miwa A, Okado H (2001) Spine formation and correlated assembly of presynaptic and postsynaptic molecules. J Neurosci 21:6105-6114.

Ramon y Cajal R (1894) La fine structure des centres nerveux. Proc R Soc Lond B Biol Sci 243-245.

Rees RP, Bunge MB, Bunge RP (1976) Morphological changes in neuritic growth cone and target neuron during synaptic junction development in culture. J Cell Biol 68:240-263.

Richards DA, Mateos JM, Hugel S, de Paola V, Caroni P, Gahwiler BH, McKinney RA (2005) Glutamate induces the rapid formation of spine head protrusions in hippocampal slice cultures. Proc Natl Acad Sci USA 102:6166-6171.

Sorra KE, Harris KM (1998) Stability in synapse number and size at $2 \mathrm{hr}$ after long-term potentiation in hippocampal area CA1. J Neurosci 18:658-671.

Toni N, Buchs PA, Nikonenko I, Bron CR, Muller D (1999) LTP promotes formation of multiple spine synapses between a single axon terminal and a dendrite. Nature 402:421-425.

White EL, Hersch SM (1982) A quantitative study of thalamocortical and other synapses involving the apical dendrites of corticothalamic projection cells in mouse Smi cortex. J Neurocytol 11:137-157.

Ziv NE, Smith SJ (1996) Evidence for a role of dendritic filopodia in synaptogenesis and spine formation. Neuron 17:91-102. 